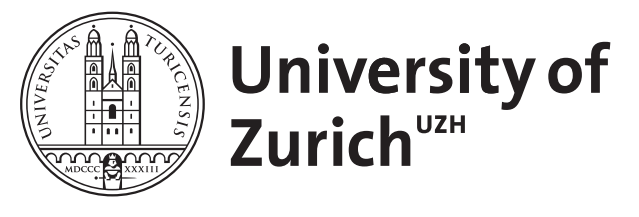

\title{
Intestinal dendritic cells
}

\author{
Schiavi, Elisa ; Smolinska, Sylwia ; O’Mahony, Liam
}

\begin{abstract}
PURPOSE OF REVIEW The intestinal immune system is constantly exposed to foreign antigens, which for the most part should be tolerated, but the immune system retains the ability to react rapidly and effectively to eliminate pathogens. Dendritic cells are at the front line in maintaining intestinal integrity as they are widely distributed within the intestinal lamina propria, Peyer's patches and mesenteric lymph nodes. RECENT FINDINGS The identification of dendritic cell subsets and phenotypic markers within the healthy and diseased intestine has progressed significantly, including improved identification of dendritic cell subsets within the human intestine. Recently, the role for dietary factors and the microbiome in modulating the intestinal dendritic cell functions has begun to be better investigated, resulting in a number of new findings relating to retinoic acid metabolism, pattern recognition receptor triggering and G-protein-coupled receptor activation. In addition, the interactions between goblet cells and mucin with intestinal dendritic cells are being better defined. SUMMARY In this review, we discuss the recent findings relating to intestinal dendritic cells, in particular the importance of dendritic cells in sensing the intestinal microenvironment and the consequences for health and disease.
\end{abstract}

DOI: https://doi.org/10.1097/MOG.0000000000000155

Posted at the Zurich Open Repository and Archive, University of Zurich

ZORA URL: https://doi.org/10.5167/uzh-118304

Journal Article

Published Version

Originally published at:

Schiavi, Elisa; Smolinska, Sylwia; O’Mahony, Liam (2015). Intestinal dendritic cells. Current Opinion in Gastroenterology, 31(2):98-103.

DOI: https://doi.org/10.1097/MOG.0000000000000155 


\title{
Intestinal dendritic cells
}

\author{
Elisa Schiavi ${ }^{\mathrm{a}}$, Sylwia Smolinska ${ }^{\mathrm{a}, \mathrm{b}, \mathrm{c}}$, and Liam O'Mahony ${ }^{\mathrm{a}}$
}

\begin{abstract}
Purpose of review
The intestinal immune system is constantly exposed to foreign antigens, which for the most part should be tolerated, but the immune system retains the ability to react rapidly and effectively to eliminate pathogens. Dendritic cells are at the front line in maintaining intestinal integrity as they are widely distributed within the intestinal lamina propria, Peyer's patches and mesenteric lymph nodes.
\end{abstract}

\section{Recent findings}

The identification of dendritic cell subsets and phenotypic markers within the healthy and diseased intestine has progressed significantly, including improved identification of dendritic cell subsets within the human intestine. Recently, the role for dietary factors and the microbiome in modulating the intestinal dendritic cell functions has begun to be better investigated, resulting in a number of new findings relating to retinoic acid metabolism, pattern recognition receptor triggering and G-protein-coupled receptor activation. In addition, the interactions between goblet cells and mucin with intestinal dendritic cells are being better defined.

\section{Summary}

In this review, we discuss the recent findings relating to intestinal dendritic cells, in particular the importance of dendritic cells in sensing the intestinal microenvironment and the consequences for health and disease.

Keywords

dendritic cells, goblet cells, microbiome, retinoic acid, short-chain fatty acids

\section{INTRODUCTION}

The intestine is a highly evolved organ specialized to perform the essential functions of nutrient digestion, absorption and waste disposal. The intestinal immune system has the momentous and unenviable task of maintaining intestinal integrity in the presence of an enormous quantity of external or foreign antigens. Highly sophisticated cellular and molecular networks need to be constantly coordinated in order to respond appropriately to such antigens, while also protective immune responses to potential pathogens must be maintained and can be induced effectively on demand. Inappropriate immune response to these antigens is a significant component in several intestinal diseases, including inflammatory bowel disease and food allergies. Dendritic cells are professional antigen-presenting cells that are present within all tissues exposed to the outside world as immature sentinel cells that efficiently sample their environment for foreign antigens $[1,2]$. In addition to their essential role in antigen presentation, dendritic cells express multiple pattern recognition receptors, which modulate their maturation resulting in the development of different dendritic cell subsets that selectively promote polarized lymphocyte responses. Upon activation, dendritic cells undergo maturation into potent $\mathrm{T}$-cell stimulatory effector or regulatory dendritic cells and migrate toward the $\mathrm{T}$ cell areas of draining lymphoid organs. There, dendritic cells will activate naive $\mathrm{T}_{\mathrm{H}}$ cells with antigen-specific [major histocompatibility complex (MHC) peptide complexes, signal 1] and costimulatory or inhibitory (signal 2) molecules. Recently, CD83 expression by intestinal dendritic cells was shown to regulate dendritic cell activation and promote mucosal homeostasis [3"]. In addition to signals 1 and 2, dendritic cells carry a third signal, which determines the polarization of naive $\mathrm{T}_{\mathrm{H}}$ cells into $\mathrm{T}_{\mathrm{H}} 1, \mathrm{~T}_{\mathrm{H}} 2, \mathrm{~T}_{\mathrm{H}} 9, \mathrm{~T}_{\mathrm{H}} 17$ or $\mathrm{T}$ regulatory cells [4]. Like signal 2, signal 3 is heterogeneous and can be mediated by various soluble or membrane-bound

\footnotetext{
${ }^{a}$ Swiss Institute of Allergy and Asthma Research, University of Zurich, Davos, Zurich, Switzerland, ${ }^{\mathrm{b}}$ ALL-MED Medical Research Institute and ${ }^{\mathrm{c} D e p a r t m e n t}$ of Clinical Immunology, Wroclaw Medical University, Wroclaw, Poland

Correspondence to Dr Liam O'Mahony, SIAF, Obere Strasse 22, 7270 Davos Platz, Switzerland. Tel: +41 81 4100853; fax: +41 81 4100840; e-mail: liam.omahony@siaf.uzh.ch
}

Curr Opin Gastroenterol 2015, 31:98-103

DOI:10.1097/MOG.0000000000000155 


\section{KEY POINTS}

- Intestinal dendritic cells are key players in mediating mucosal regulatory responses, effector responses and pathological responses.

- The intestinal environment, including the microbiome and dietary factors, shape and condition intestinal dendritic cell activity.

- Goblet cells and mucins are involved in intestinal dendritic cell antigen sampling and modulation of dendritic cell activity.

molecules, including interleukin (IL)-1 $\beta$, IL-6, IL-10, IL-12, IL-18, TGF- $\beta$, IFN- $\alpha$, OX40 ligand (OX40L) and retinoic acid $[5,6]$. Importantly, in-vitro studies suggest that the expression levels of these $\mathrm{T}_{\mathrm{H}}$-cellpolarizing molecules by mature dendritic cells strongly depend on the conditions during their initial activation as sentinel dendritic cells. These findings imply that pathogens, commensals and dietary components may promote the development of distinct dendritic cell phenotypes by provoking tissues to release mediators involved in polarization. The interplay between immunostimulatory and suppressive activities of dendritic cells is important for both the induction of an immune response and the maintenance of local immunostasis. The recent findings regarding the environmental factors influencing dendritic cell polarization will be discussed in the following review.

\section{DENDRITIC CELL SUBSETS WITHIN THE INTESTINE}

Intestinal dendritic cells are located within specific intestinal lymphoid tissues, collectively termed gutassociated lymphoid tissues (GALT), or diffusely distributed throughout the intestinal lamina propria [7]. Dendritic cell subsets are not evenly distributed along the intestinal tract, rather the $\mathrm{CD} 103^{+}$ $\mathrm{CD}_{11 \mathrm{~b}^{+}}$dendritic cells are the major dendritic cell subset in the murine small intestine lamina propria, whereas in the colon these cells are reduced, resulting in higher proportions of $\mathrm{CD}_{103}{ }^{+} \mathrm{CD} 11 \mathrm{~b}^{-}$and $\mathrm{CD} 103^{-} \mathrm{CD} 11 \mathrm{~b}^{+}$dendritic cells [8]. Dendritic cell subsets in the intestine have been extensively studied in mice, but studies in humans have been complicated by the realization that murine markers do not always correlate with human markers. Recently, a comparison between human and murine gut dendritic cell populations revealed that human $\mathrm{CD} 103^{+}$signal regulatory protein alpha $(\operatorname{Sirp} \alpha)^{-}$ intestinal dendritic cells were related to murine intestinal $\mathrm{CD} 103^{+} \mathrm{CD} 11 \mathrm{~b}^{-}$dendritic cells. Human
$\mathrm{CD}_{103}{ }^{+} \mathrm{Sirp} \alpha^{+}$dendritic cells are closely related to murine intestinal $\mathrm{CD}_{103}{ }^{+} \mathrm{CD} 11 \mathrm{~b}^{+}$dendritic cells and they supported the induction of regulatory $\mathrm{T}$ cells. Both human $\mathrm{CD} 103^{+}$dendritic cell subsets induced $\mathrm{T}_{\mathrm{H}} 17$ polarization, whereas CD103-Sirp $\alpha^{+}$ dendritic cells promoted $\mathrm{T}_{\mathrm{H}} 1$ polarization [9"']. These authors also performed comparative transcriptomics, which identified gene clusters that are evolutionarily conserved or divergent between mice and humans. The importance of SIRP $\alpha$ in the homeostasis of $\mathrm{CD}_{103}{ }^{+} \mathrm{CD} 11 \mathrm{~b}^{+}$dendritic cells in the intestine was recently demonstrated, in which loss of SIRP $\alpha$ signaling in mice leads to a selective reduction in the $\mathrm{CD} 103^{+} \mathrm{CD} 11 \mathrm{~b}^{+}$dendritic cells within the mucosa associated with reduced numbers of $\mathrm{T}_{\mathrm{H}} 17$ cells in steady-state intestinal mucosa and a defective $\mathrm{T}_{\mathrm{H}} 17$ response to Citrobacter rodentium infection [10"]. The CD103- dendritic cell subset has been associated with colitis severity and osteopontin expression by $\mathrm{CD}^{-103^{-}}$dendritic cells drives intestinal inflammation. Disrupting the interaction between osteopontin and integrin $\alpha 9$ suppressed the inflammatory potential of CD103- dendritic cells in vitro and in vivo [11"].

\section{DENDRITIC CELL MODULATION BY MICROBES AND MICROBIAL METABOLITES}

The balance between immune tolerance and inflammation is regulated through the crosstalk between innate immune cells and the intestinal microbiota involving many signaling pathways and molecules. One component of these regulatory activities includes host-microbe-metabolic interactions and involves many organs, including the gut [12]. Intestinal dendritic cells are one of the main targets for immunomodulatory processes related to commensal microbes [13]. Metabolism of vitamin A to retinoic acid is a key immunomodulatory activity associated with intestinal dendritic cells [14"]. Certain, but not all, commensal microbes can induce retinoic acid metabolism by human dendritic cells in vitro and by murine $\mathrm{CD}_{103}{ }^{+}$dendritic cells within the small intestine lamina propria $\left[15^{*}, 16\right]$. Retinoic acid has also recently been described to play a role in immune regulation within the gastric mucosa. Gastric epithelial cells were described to promote retinoic acid metabolism in dendritic cells from the gastric mucosa. Helicobacter pylori infection severely disrupted gastric retinoic acid biosynthesis, which may lead to reduced dendritic cell retinoic acid signaling and may contribute to disease progression [17"]. In addition to vitamin A metabolism, induction of another dendritic cell metabolic enzyme, heme oxygenase-1 (HO-1), was shown to be required for the 
induction of mucosal T regulatory cells within mesenteric lymph nodes by Lactobacillus rhamnosus. Upregulation of dendritic cell HO-1 resulted from direct contact with L. rhamnosus [18].

Bacterial cell wall components and metabolites [including cell-surface-associated or secreted polysaccharides, short-chain fatty acids (SCFAs), vitamins and biogenic amines, e.g., histamine] have been associated with immunoregulatory effects on dendritic cells at mucosal interfaces [19]. There is also evidence that these products can shape the immune response not only locally but also at extraintestinal sites $\left[2^{*}\right]$, and they can act not only on dendritic cells but also directly on T cells, macrophages, invariant natural killer T cells and epithelial cells [21-23]. However, dendritic cells are the primary cell type involved as 'sensors' of microbial ligands through the activation of innate immune receptors (e.g. Toll-like receptors and c-type lectin receptors) expressed on their cell surface or inside the cytoplasm. The signaling pathways triggered by commensal-derived molecules allow for changes in dendritic cell phenotypes and cytokine secretion, which underlie the integration of microbial and host metabolism with immune functions.

Structural components of commensal microbes have been shown to be important for dendritic cell sensing of luminal bacterial species. For example, MHC-II-dependent antigen presentation of segmented filamentous bacteria antigens by intestinal $\mathrm{CD}_{11 \mathrm{c}^{+}}$dendritic cells is crucial for the local induction of $\mathrm{T}_{\mathrm{H}} 17$ lymphocytes [24"-]. In addition, a variety of polysaccharides from bacterial cell walls have been described to influence dendritic cell function. Capsular polysaccharide A (PSA) from the gut bacterium Bacteroides fragilis has been associated with regulatory properties. Its mechanism of action is not yet fully clear, but this polysaccharide has been shown to interact directly with mouse plasmacytoid dendritic cells via TLR-2. PSA-exposed plasmacytoid dendritic cells express molecules involved in protection against colitis and stimulated $\mathrm{CD} 4^{+}$ cells to secrete IL-10 [25"]. In a multiple sclerosis mouse model, the same ligand protected against central nervous system demyelination and inflammation via TLR-2, and one of the possible mechanisms underlying these effects could involve dendritic cells [26]. PSA also interacts with human dendritic cell-specific Intercellular adhesion molecule-3-grabbing non-integrin (DC-SIGN) receptor expressed on monocyte-derived dendritic cells, which endocytose PSA thus facilitating presentation to $\mathrm{T}$ cells and the proliferation of PSA-specific CD4 ${ }^{+}$ lymphocytes [27"]. Exopolysaccharides (EPS) which can be secreted or associated with the bacteria cell wall have also received attention for their potential tolerogenic properties. EPS from Bacillus subtilis is able to prevent gut inflammation stimulated by C. rodentium, which is dependent on TLR-4 and MyD88 signaling in mouse myeloid cells including macrophages and dendritic cells [28"]. $\beta$-Glucans, which are polysaccharides found in bacteria, fungi and yeasts, are molecules which can have proinflammatory and anti-inflammatory properties and they have been studied for their potential benefits in cancer treatment and infection control. $\beta$-Glucan from Saccharomyces cerevisiae triggers murine dendritic cells to secrete TNF- $\alpha$, IL-10 and TGF- $\beta$. The production of these cytokines as well as stimulation of indoleamine 2,3-dioxygenase (IDO) enzyme expression is dependent on Dectin-1 receptor engagement [29]. A population of Langerin-positive dendritic cells in murine Peyer's patches was demonstrated to be involved in sampling $\beta$-glucan microparticles following transepithelial transport via M cells [30"]. Certain carbohydrate structures can have dual effects on dendritic cells and one example is the mannose-capped lipoarabinomannan (Man-LAM) from Mycobacterium tuberculosis. This lipoglycan has the ability to induce both proinflammatory (TNF, IL-6 and IL-12p40) and antiinflammatory (IL-10) cytokines by dendritic cells in a Dectin-2-dependent manner [31]. Lastly, dietaryderived oligosaccharides can directly influence mucosal dendritic cells. The breast milk oligosaccharide sialyl $(\alpha 2,3)$ lactose directly stimulated mesenteric lymph node $\mathrm{CD} 11 \mathrm{c}^{+}$dendritic cells to secrete cytokines required for the expansion of $\mathrm{T}_{\mathrm{H}} 1$ and $\mathrm{T}_{\mathrm{H}} 17 \mathrm{~T}$ cells. The stimulatory effect was attenuated in Tlr4-deficient CD11 ${ }^{+}$cells, demonstrating that this oligosaccharide induces inflammation through TLR-4 signaling [32"'].

Recently, significant attention has been focused on the immunoregulatory role for SCFAs produced by commensal microbes within the gut. Species of Roseburia, Eubacterium, Bacteroides and Faecalibacterium are examples of bacteria that produce these metabolites in the gut ecosystem. The production of SCFAs occurs in the colon following fermentation of dietary fibers [33]. Abnormalities in the production of these metabolites (because of dietary factors and dysbiosis) might play a role in the pathogenesis of type 2 diabetes, obesity, inflammatory bowel disease, colorectal cancer and allergies [34]. Among the SCFAs, butyrate seems to be more potent than acetate or propionate in inducing immunomodulatory effects within the gut. Butyrate influences histone deacetylases (HDAC) activity, which is responsible for decreasing dendritic cell proinflammatory cytokine production (IL-12 and IL-6) and allows dendritic cells to promote $T$ regulatory cells. Propionate is less potent than butyrate, although 
propionate can also contribute to the induction of Foxp3 expression by dendritic cells, whereas acetate does not have this activity possibly because of the lack of HDAC activity [35"]. HDAC inhibition by butyrate has been also shown for intestinal macrophages [36]. Another recent study has confirmed and extended the observation that butyrate promotes dendritic cell regulatory activity, resulting in the induction of $\mathrm{T}$ regulatory cells and IL-10producing $\mathrm{T}$ cells. These effects were mediated by the G-protein coupled receptor Gpr109a on colonic dendritic cells and macrophages, whereas Gpr109adeficient mice were susceptible to the development of colonic inflammation and colon cancer [37"].

Histamine is another important mucosal metabolite and gut histamine levels are increased in patients with irritable bowel syndrome and inflammatory bowel disease [38"]. Histamine is able to decrease chemokine and proinflammatory cytokine secretion induced by Toll-like receptor stimulated dendritic cells, while increasing IL-10 production [39"']. Histamine exerted this effect by activating the histamine 2 receptor $\left(\mathrm{H}_{2} \mathrm{R}\right)$ on dendritic cells, and the signaling mechanism required cAMP and exchange protein directly activated by cAMP (EPAC). Histamine is secreted not only by the host immune cells, but also by the bacteria within the gut. Administration of a histamine-secreting Lactobacillus strain to mice resulted in rapid weight loss and enhanced Peyer's patch cytokine secretion, which was exaggerated in $\mathrm{H}_{2} \mathrm{R}$-deficient animals [40"].

\section{DENDRITIC CELL INTERACTIONS WITH GOBLET CELLS AND MUCINS}

The gastrointestinal tract is covered by a mucus layer that has different properties in the stomach, small intestine and colon. In the small intestine, mucus limits the number of bacteria that can reach the epithelium and the Peyer's patches. In the large intestine, the inner mucus layer separates the commensal bacteria from the host epithelium. The outer colonic mucus layer is the natural habitat for the commensal bacteria. Mucus is secreted by goblet cells and typically contains several major components. One of these, the mucins gives it the mucus gel-like properties. The mucins produced by goblet cells are the classical gel-forming mucins MUC2, MUC5AC, MUC6 and MUC5B, whereas the transmembrane mucins MUC1, MUC3, MUC4, MUC12, MUC13 and MUC17 are all found in the gastrointestinal tract [41].

Previously, the function of goblet cells was thought to fulfill a purely secretory role within the intestine. However, recent evidence suggests that goblet cells in the small intestine deliver luminal antigens to the dendritic cells within the lamina propria [42]. The uptake of luminal material by goblet cells is stimulated by acetyl choline analogs acting on

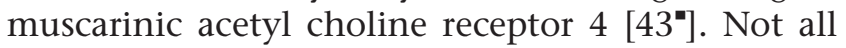
dendritic cell subsets sampled antigen from goblet cells, the $\mathrm{CD} 11 \mathrm{c}^{+} \mathrm{CD} 103^{+}$dendritic cell subpopulation seems to be uniquely capable of performing this role. These dendritic cells can also cross-present antigens to $\mathrm{T}$ cells and induce tolerance, as described above. However, the mechanisms underpinning the goblet cells and $\mathrm{CD} 103^{+}$dendritic cells interactions that cooperate to promote tolerance is far from being understood, but it does seem that goblet cells have a new type of gate-keeping role for the presentation of oral antigen to dendritic cells within the lamina propria.

Mucin secreted by the goblet cells has also been recently shown to influence intestinal dendritic cell activity [44"']. Dendritic cells take up MUC2 and glycans associated with MUC2 inhibited dendritic cell inflammatory responses, but not tolerogenic responses, by inducing a galectin-3-Dectin-1Fc $\gamma$ RIIB receptor complex that activated $\beta$-catenin, resulting in nuclear factor kappa-light-chainenhancer of activated B cells (NF-kB) inhibition. Thus, mucus not only merely forms a nonspecific physical barrier, but also constrains the immunogenicity of gut antigens by delivering tolerogenic signals to mucosal dendritic cells.

\section{CONCLUSION}

The interplay between immunostimulatory and suppressive activities of intestinal dendritic cells is important for both the induction of an immune response and the maintenance of local immunostasis. The continuing identification of dendritic cell subsets within the human intestine and the elucidation of signaling mechanisms that govern mucosal dendritic cell activity are crucial to better understand the aberrant immune responses associated with a range of gastrointestinal disorders, including inflammatory bowel disease, irritable bowel syndrome and food allergy. In addition, the recent exciting discoveries describing the factors that mediate microbiome-host dialog may provide new candidate molecules for the deliberate modulation of dendritic cell activity.

\section{Acknowledgements}

None.

\section{Financial support and sponsorship}

The authors are supported by the Swiss National Foundation grants (project numbers: 310030-127356 and 
310030-144219), Allergiestiftung Ulrich Müller-Gierok, European Union Marie Curie grants and Polish National Science Centre grant no. 2011/01/B/NZ6/01872, 2012/ 04/M/NZ6/00355 and 2012/04/A/NZ6/00407. L.O.M. is a consultant to Alimentary Health Ltd. and has received research funding from GSK.

\section{Conflicts of interest}

\section{E.S. and S.S. have no conflicts of interest.}

\section{REFERENCES AND RECOMMENDED READING}

Papers of particular interest, published within the annual period of review, have been highlighted as:

- of special interest

-1 of outstanding interest

1. Persson EK, Scott CL, Mowat AM, Agace WW. Dendritic cell subsets in the intestinal lamina propria: ontogeny and function. Eur J Immunol 2013; 43:3098-3107.

2. Chang SY, Ko HJ, Kweon MN. Mucosal dendritic cells shape mucosal immunity. Exp Mol Med 2014; 46:e84.

3. Bates JM, Flanagan $\mathrm{K}, \mathrm{Mo} \mathrm{L}$, et al. Dendritic cell CD83 homotypic interactions

- regulate inflammation and promote mucosal homeostasis. Mucosal Immunol 2014. [Epub ahead of print]

These authors show that CD83 expression by dendritic cells prevents colitis.

4. Kalinski $P$, Hilkens CMU, Wierenga EA, Kapsenberg ML. T-cell priming by type- 1 and type-2 polarized dendritic cells: the concept of a third signal. Immunol Today 1999; 20:561-567.

5. Akdis M, Burgler S, Crameri R, et al. Interleukins, from 1 to 37 , and interferon$\gamma$ : receptors, functions, and roles in diseases. J Allergy Clin Immunol 2011; 127:701-721.

6. Coombes JL, Siddiqui KR, Arancibia-Cárcamo CV, et al. A functionally specialized population of mucosal $\mathrm{CD}_{103^{+}} \mathrm{DC}$ s induces $\mathrm{Foxp} 3^{+}$regulatory T cells via a TGF-beta and retinoic acid-dependent mechanism. J Exp Med 2007; 204:1757-1764

7. Bekiaris V, Persson EK, Agace WW. Intestinal dendritic cells in the regulation of mucosal immunity. Immunol Rev 2014; 260:86-101.

8. Denning TL, Norris BA, Medina-Contreras $\mathrm{O}$, et al. Functional specializations of intestinal dendritic cell and macrophage subsets that control Th17 and regulatory $\mathrm{T}$ cell responses are dependent on the T cell/APC ratio, source of mouse strain, and regional localization. J Immunol 2011; 187:733-747.

9. Watchmaker PB, Lahl $\mathrm{K}$, Lee $\mathrm{M}$, et al. Comparative transcriptional and

n. functional profiling defines conserved programs of intestinal DC differentiation in humans and mice. Nat Immunol 2014; 15:98-108.

This report provides a comprehensive description of murine and human intestinal dendritic cell subsets, and highlights the evolutionarily conserved and divergent programming of intestinal dendritic cells.

10. Scott CL, Tfp ZM, Beckham KS, et al. Signal regulatory protein alpha (SIRP $\alpha)$

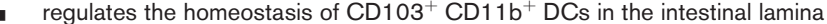
propria. Eur J Immunol 2014; 44:3658-3668.

This study shows a previously unappreciated role for SIRP $\alpha$ in the homeostasis of CD $103^{+}$CD $11 b^{+}$dendritic cells in the intestine and provides further evidence that this dendritic cell subset is involved in the development of mucosal $T_{H} 17$ responses.

11. Kourepini $E$, Aggelakopoulou $M$, Alissafi $T$, et al. Osteopontin expression by $\mathrm{CD}_{103}{ }^{-}$dendritic cells drives intestinal inflammation. Proc Natl Acad Sci USA 2014; 111 :E856-865.

This report contributes additional key information into the biology of CD103dendritic cells and their function during inflammatory bowel disease.

12. Sommer F, Bäckhed F. The gut microbiota - masters of host development and physiology. Nat Rev Microbiol 2013; 11:227-238.

13. McDermott AJ, Huffnagle GB. The microbiome and regulation of mucosal immunity. Immunology 2014; 142:24-31.

14. Bakdash G, Vogelpoel $L T$, van Capel TM, et al. Retinoic acid primes human

- dendritic cells to induce gut-homing, IL-10-producing regulatory $\mathrm{T}$ cells. Mucosal Immunol 2014. [Epub ahead of print]

This study shows that retinoic acid induces IL-10-producing $T$ cells and postulates a novel mechanism for IL-10 in maintaining tolerance to the intestinal microbiome.

15. Konieczna $P$, Ferstl $R$, Ziegler $M$, et al. Immunomodulation by Bifidobacterium

- infantis 35624 in the murine lamina propria requires retinoic acid-dependent and independent mechanisms. PLoS One 2013; 8:e62617.

This study describes the induction of retinoic acid metabolism in $\mathrm{CD}_{103^{+}}$ dendritic cells by a specific microbial species within the gut.
16. Konieczna $P$, Groeger D, Ziegler M, et al. Bifidobacterium infantis 35624 administration induces Foxp3 T regulatory cells in human peripheral blood: potential role for myeloid and plasmacytoid dendritic cells. Gut 2012;61:354366.

17. Bimczok D, Kao JY, Zhang M, et al. Human gastric epithelial cells contribute to gastric immune regulation by providing retinoic acid to dendritic cells. Mucosal Immunol 2014. [Epub ahead of print]

This report shows a novel role for retinoic acid in human gastric immune regulation.

18. Karimi K, Kandiah N, Chau J, et al. A Lactobacillus rhamnosus strain induces a heme oxygenase dependent increase in Foxp $3^{+}$regulatory T cells. PLoS One 2012; 7:e47556.

19. Frei R, Lauener RP, Crameri R, O'Mahony L. Microbiota and dietary interactions: an update to the hygiene hypothesis? Allergy $2012 ; 67: 451-$ 461.

20. Trompette A, Gollwitzer ES, Yadava K, et al. Gut microbiota metabolism of

- dietary fiber influences allergic airway disease and hematopoiesis. Nat Med $2014 ; 20: 159-166$.

This study shows that dietary fermentable fiber and SCFAs can shape the immunological environment and influence the severity of allergic inflammation.

21. Maslowski KM, Vieira AT, Ng A, et al. Regulation of inflammatory responses by gut microbiota and chemoattractant receptor GPR43. Nature 2009; 461: $1282-1286$.

22. An $D$, Oh SF, Olszak T, et al. Sphingolipids from a symbiotic microbe regulate homeostasis of host intestinal natural killer T cells. Cell 2014; 156:123-133.

23. Atarashi $\mathrm{K}$, Tanoue $\mathrm{T}$, Oshima $\mathrm{K}$, et al. Treg induction by a rationally selected mixture of Clostridia strains from the human microbiota. Nature 2013; $500: 232-236$

24. Goto $\mathrm{Y}$, Panea $\mathrm{C}$, Nakato $\mathrm{G}$, et al. Segmented filamentous bacteria antigens

I. presented by intestinal dendritic cells drive mucosal Th17 cell differentiation. Immunity 2014; 40:594-607.

This report describes the complex role of dendritic cells and innate lymphoid cells in the regulation of intestinal Th17 cell homeostasis in response to a gut microbe.

25. Dasgupta S, Erturk-Hasdemir D, Ochoa-Reparaz J, et al. Plasmacytoid

- dendritic cells mediate anti-inflammatory responses to a gut commensal molecule via both innate and adaptive mechanisms. Cell Host Microbe $2014 ; 15: 413-423$

This study shows that plasmacytoid dendritic cells can orchestrate the beneficial immunoregulatory interaction of commensal microbial molecules via both innate and adaptive immune mechanisms.

26. Wang $Y$, Telesford $K M$, Ochoa-Repáraz J, et al. An intestinal commensal symbiosis factor controls neuroinflammation via TLR2-mediated CD39 signalling. Nat Commun 2014; 5:4432.

27. Bloem K, García-Vallejo JJ, Vuist IM, et al. Interaction of the capsular poly-

- saccharide A from Bacteroides fragilis with DC-SIGN on human dendritic cells is necessary for its processing and presentation to T cells. Front Immunol 2013; 4:103

These authors describe a crucial role for DC-SIGN in the endocytosis and routing of capsular polysaccharide $A$ in human dendritic cells for the efficient stimulation of lymphocytes.

28. Jones SE, Paynich ML, Kearns DB, Knight KL. Protection from intestinal inflammation by bacterial exopolysaccharides. J Immunol 2014; 192:48134820 .

This study demonstrates that bacterial exopolysaccharides prevent colitis in a TLR4-dependent manner, which requires myeloid cells.

29. Karumuthil-Melethil $S$, Gudi $R$, Johnson BM, et al. Fungal $\beta$-glucan, a dectin-1 ligand, promotes protection from type 1 diabetes by inducing regulatory innate immune response. J Immunol 2014; 193:3308-3321.

30. De Jesus M, Ostroff GR, Levitz SM, et al. A population of Langerin-positive

- dendritic cells in murine Peyer's patches involved in sampling $\beta$-glucan microparticles. PLoS One 2014; 9:e91002.

This study shows that microparticles are sampled by a population of dendritic cells within the Peyer's patches that express Langerin.

31. Yonekawa A, Saijo S, Hoshino $Y$, et al. Dectin-2 is a direct receptor for mannose-capped lipoarabinomannan of mycobacteria. Immunity 2014; 41: 402-413.

32. Kurakevich $E$, Hennet $T$, Hausmann $M$, et al. Milk oligosaccharide sialy-

- I $(\alpha 2,3)$ lactose activates intestinal $C_{1} 11 c^{+}$cells through TLR4. Proc Natl Acad Sci USA 2013; 110:17444-17449.

These authors demonstrate a novel mechanism by which breast milk oligosaccharides shape the intestinal environment by affecting mucosal immunity.

33. Tan J, McKenzie C, Potamitis $M$, et al. The role of short-chain fatty acids in health and disease. Adv Immunol 2014; 121:91-119.

34. Thorburn AN, Macia L, Mackay CR. Diet, metabolites, and 'western-lifestyle' inflammatory diseases. Immunity 2014; 40:833-842.

35. Arpaia N, Campbell C, Fan X, et al. Metabolites produced by commensal

- bacteria promote peripheral regulatory T-cell generation. Nature 2013; 504:451-455

This study shows that butyrate, produced by commensal microorganisms, facilitated extrathymic generation of $\mathrm{T}$ regulatory cells.

36. Chang PV, Hao L, Offermanns S, Medzhitov R. The microbial metabolite butyrate regulates intestinal macrophage function via histone deacetylase inhibition. Proc Natl Acad Sci USA 2014; 111:2247-2252. 
37. Singh N, Gurav A, Sivaprakasam S, et al. Activation of Gpr109a, receptor for - niacin and the commensal metabolite butyrate, suppresses colonic inflammation and carcinogenesis. Immunity 2014; 40:128-139.

This study demonstrates that Gpr109a has an essential role in mediating the beneficial effects of gut microbiota and dietary fiber in colon.

38. Smolinska S, Jutel M, Crameri R, O'Mahony L. Histamine and gut mucosal - immune regulation. Allergy 2014; 69:273-281.

This report comprehensively describes the cellular sources and immunological effects of histamine within the gut.

39. Frei R, Ferstl R, Konieczna $\mathrm{P}$, et al. Histamine receptor 2 modifies dendritic cell mesponses to microbial ligands. J Allergy Clin Immunol 2013; 132:194-204. This study describes the immunoregulatory molecular mechanisms associated with histamine modulation of dendritic cell activation following TLR stimulation.

40. Ferstl R, Frei R, Schiavi E, et al. Histamine receptor 2 is a key influence in immune

- responses to intestinal histamine-secreting microbes. J Allergy Clin Immunol $2014 ; 134: 744-746$

This report highlights the immunoregulatory role for histamine secreted by gut microbes.
41. Pelaseyed $\mathrm{T}$, Bergström JH, Gustafsson JK, et al. The mucus and mucins of the goblet cells and enterocytes provide the first defense line of the gastrointestinal tract and interact with the immune system. Immunol Rev 2014; 260:8-20

42. McDole JR, Wheeler LW, McDonald KG, et al. Goblet cells deliver luminal antigen to $\mathrm{CD}_{103^{+}}$dendritic cells in the small intestine. Nature 2012; 483:345-349.

43. Knoop KA, McDonald KG, McCrate $S$, et al. Microbial sensing by goblet cells

- controls immune surveillance of luminal antigens in the colon. Mucosal Immunol 2015; 8:198-210.

These authors describe that goblet cells play a critical role in regulating the exposure of the colonic immune system to luminal antigens.

44. Shan M, Gentile M, Yeiser JR, et al. Mucus enhances gut homeostasis and

- oral tolerance by delivering immunoregulatory signals. Science 2013; 342:447-453

This study demonstrates that mucus not only merely forms a nonspecific physical barrier, but also constrains the immunogenicity of gut antigens by delivering tolerogenic signals to mucosal dendritic cells. 